\title{
Analysis of Stability of a Plasma in Porous Medium
}

\author{
PARDEEP KUMAR \\ Department of Mathematics, ICDEOL, Himachal Pradesh University, Summerhill, \\ Shimla-5, INDIA
}

\begin{abstract}
The thermal convection of a plasma in porous medium is investigated in the presence of finite Larmor radius (FLR) and Hall effects. Following linear stability theory and normal mode analysis method, the dispersion relation is obtained. It is found that the presence of a magnetic field (and hence the presence of FLR and Hall effects) introduces oscillatory modes in the system which were, otherwise, non-existent in their absence. When the instability sets in as stationary convection, the FLR may have a stabilizing or destabilizing effect, but a completely stabilizing one for a certain wave-number range. Similarly, the Hall currents may have a stabilizing or destabilizing effect but a completely stabilizing one for the same wave-number range under certain condition, whereas the medium permeability always has a destabilizing effect for stationary convection. Also it is found that the system is stable for $\frac{g \alpha \kappa}{v \beta} \leq \frac{27 \pi^{4}}{4}$ and under the condition $\frac{g \alpha \kappa}{v \beta}>\frac{27 \pi^{4}}{4}$, the system becomes unstable.
\end{abstract}

Key-words: Finite Larmor Radius Effect, Hall Effects, Plasma, Porous Medium, Thermal Convection

Received: May 25, 2021. Revised: November 22, 2021. Accepted: December 18, 2021. Published: January 5, 2022.

\section{Introduction}

The theoretical and experimental results on thermal convection in a fluid layer, under varying assumptions of hydrodynamics and hydromagnetics, have been discussed in a treatise by Chandrasekhar [1]. The effects of the finiteness of the ion Larmor radius which exhibits itself in the form of 'magnetic viscosity' in the fluid equations have been studied by many authors (Jukes [2]; Vandakurov [3]). Sharma and Prakash [4] have studied the effect of finite Larmor radius on the thermal instability of a plasma. Melchior and Popowich [5] have considered the finite Larmor radius effect on the Kelvin-Helmholtz instability in a fully ionized plasma while that on Rayleigh-Taylor instability has been studied by Singh and Hans [6]). The effect of finite Larmor radius on the thermal instability of a plasma in the presence of a vertical magnetic field has been studied by Sharma [7].

The study of the breakdown of the stability of a layer of fluid subject to a vertical temperature gradient in porous medium and the possibility of convective flow is of considerable interest in recent years. The study of onset of convection in a porous medium has attracted considerable interest because of its natural occurrence and of its intrinsic importance in many industrial problems, particularly in petroleum-exploration, chemical and nuclear industries. The derivation of the basic equations of a layer of fluid heated from below in porous medium, using Boussinesq approximation, has been given by Joseph [8]. The study of a layer of fluid heated from below in porous media is motivated both theoretically and by its practical applications in engineering disciplines. Among the applications in engineering disciplines one can find the food process industry, chemical process industry, solidification and centrifugal casting of metals. The development of geothermal power resources has increased general interest in the properties of convection in porous medium. Generally it is accepted that comets consists of a dusty 'snowball' of a mixture of frozen gases which in the process of their journey changes from solid to gas and vice-versa. The physical properties of comets, meteorites and interplanetary dust strongly suggest the importance of porosity in the astrophysical context (McDonnel [9]). The effect of a magnetic field on the stability of such a flow is of interest in geophysics, particularly in the study of the Earth's core where the Earth's 
mantle, which consists of a conducting fluid, behaves like a porous medium which can become convectively unstable as a result of differential diffusion. The other application of the results of flow through a porous medium in the presence of a magnetic field is in the study of the stability of a convective flow in the geothermal region. Lapwood [10] has studied the stability of convective flow in a porous medium using Rayleigh's procedure. The Rayleigh instability of a thermal boundary layer in flow through a porous medium has been considered by Wooding [11].

The properties of ionized space and laboratory magnetic fluids (plasmas) have been intensively investigated theoretically and experimentally in the past sixty years. One of the key aspects studied in this context is the stability of plasma structures. Usually, instabilities can be divided into two categories: macro- and microinstabilities. Macro-instabilities occur with low frequencies compared to the plasma and cyclotron frequency and they are studied within the framework of magnetohydrodynamics (MHD). Physicists have understood the behaviour of macro-instabilities and they showed how to avoid the most destructive of them, but small-scale gradient driven micro-instabilities are still a serious obstacle for having a stable plasma for a large range of parameters. Microinstabilities are described by models which include, e.g. finite Larmor radius (FLR) and collision less dissipative effects in plasmas. Time and length scales of micro-instabilities are comparable to the turbulent length scales and the length scales of transport coefficients. In general, the FLR effect is neglected. However, when the Larmor radius becomes comparable to the hydromagnetic length of the problem (e.g. wavelength) or the gyration frequency of ions in the magnetic field is of the same order as the wave frequency, finiteness of the Larmor radius must be taken into account. Strictly speaking, the space and time scale for the breakdown of hydromagnetics are on the respective scales of ion gyration about the field, and the ion Larmor frequency. In the present paper, we explore the effect of FLR and Hall effects on the thermal instability of a plasma in porous medium. Finite Larmor radius effect on plasma instabilities has been the subject of many investigations. In many astrophysical plasma situations such as in solar corona, interstellar and interplanetary plasmas the assumption of zero Larmor radius is not valid. Roberts and Taylor [12] and Rosenbluth et al. [13] have shown the stabilizing influence of finite ion Larmor radius (FLR) effects on plasma instabilities. Hernegger [14] investigated the stabilizing effect of FLR on thermal instability and showed that thermal criterion is changed by FLR for wave propagation perpendicular to the magnetic field. Sharma [15] investigated the stabilizing effect of FLR on thermal instability of rotating plasma. Ariel [16] discussed the stabilizing effect of FLR on thermal instability of conducting plasma layer of finite thickness surrounded by a non-conducting matter. Vaghela and Chhajlani [17] studied the stabilizing effect of FLR on magneto-thermal stability of resistive plasma through a porous medium with thermal conduction. Bhatia and Chhonkar [18] investigated the stabilizing effect of FLR on the instability of a rotating layer of self-gravitating plasma incorporating the effects of viscosity and Hall current. Vyas and Chhajlani [19] pointed out the stabilizing effect of FLR on the thermal instability of magnetized rotating plasma incorporating the effects of viscosity, finite electrical conductivity, porosity and thermal conductivity. Kaothekar and Chhajlani [20] investigated the problem of Jeans instability of self-gravitating rotating radiative plasma with finite Larmor radius corrections. The frictional effect of collisions of ionized with neutral atoms on Rayleigh-Taylor instability of a composite plasma in porous medium has been considered by Kumar and Mohan [21]. Thus FLR effect is an important factor in the discussion of thermal convection and other hydromagnetic instabilities. Keeping these in mind, an attempt is made to study the effects of finite Larmor radius and Hall effects on the thermal convection of incompressible plasma in porous medium in the present paper.

\section{Formulation of the Problem and Perturbation Equations}

Here we consider an infinite horizontal layer of viscous, heat conducting and finite electrically conducting fluid of thickness $d$ bounded by the planes $z=0$ and $z=d$ in an isotropic and homogeneous medium of porosity $\varepsilon$ and medium permeability $k_{1}$. This layer is heated from below such that a steady temperature gradient $\beta(=$ 
$|d T / d z|)$ is maintained. Consider the cartesian coordinates $(x, y, z)$ with origin on the lower boundary $z=0$ and the $z$-axis perpendicular to it along the vertical. The fluid is acted on by a horizontal magnetic field $\vec{H}(H, 0,0)$ and gravity force $\vec{g}(0,0,-g)$.

Let $\vec{q}(u, v, w), \vec{h}\left(h_{x}, h_{y}, h_{z}\right), \delta \rho$ and $\theta$ denote respectively the perturbations in fluid (filter) velocity $(0,0,0)$, magnetic field $\vec{H}$, density $\rho$ and temperature $T$. Then the linearized hydromagnetic perturbation equations relevant to the problem are

$$
\begin{aligned}
\frac{1}{\varepsilon} \frac{\partial \vec{q}}{\partial t}=-\frac{1}{\rho_{0}} \nabla \stackrel{\leftrightarrow}{P} & -\frac{1}{\rho_{0}} \nabla \delta p-\frac{v}{k_{1}} \vec{q} \\
& +\frac{1}{4 \pi \rho_{0}}(\nabla \times \vec{h}) \times \vec{H}-\vec{g} \alpha \theta,
\end{aligned}
$$

$\nabla \cdot \vec{q}=0$

$$
\begin{gathered}
\varepsilon \frac{\partial \vec{h}}{\partial t}=\nabla \times(\vec{q} \times \vec{H})+\varepsilon \eta \nabla^{2} \vec{h}-\left(\frac{\varepsilon}{4 \pi N e}\right) \nabla \\
\times[(\nabla \times \vec{h}) \times \vec{H}],
\end{gathered}
$$

$\nabla \cdot \vec{h}=0$,

$E \frac{\partial \theta}{\partial t}=\beta w+\kappa \nabla^{2} \theta$,

where

$$
E=\varepsilon+(1-\varepsilon) \frac{\rho_{s} c_{s}}{\rho_{0} c_{v}} .
$$

Here

$$
\overleftrightarrow{P}, \rho_{0}, \mu, v\left(=\mu / \rho_{0}\right), K, \kappa(=
$$

$\left.\frac{K}{\rho_{0} c_{v}}\right), \eta, \alpha, N$ and $e$ stand for stress tensor taking into account FLR effect, density at reference level $z=0$, viscosity, kinematic viscosity, thermal conductivity, thermal diffusivity, electrical resistivity, coefficient of volume expansion, electron number density and charge of an electron respectively. $\varepsilon, k_{1}$ denote the medium porosity, medium permeability while $\rho_{0}, c_{v} ; \rho_{s}, c_{S}$ stand for density and specific heat of fluid and solid (porous matrix) material respectively. In writing (1), use has been made of the equation of state

$\rho=\rho_{0}\left[1-\alpha\left(T-T_{0}\right)\right]$,

where the suffix zero refers to values at the reference level $z=0$ and so the change in density $\delta \rho$ caused by the perturbation $\theta$ in temperature is given by

$\delta \rho=-\alpha \rho_{0} \theta$.

For the horizontal magnetic field $\vec{H}(H, 0,0)$, the stress tensor $\overleftrightarrow{P}$, taking into account the finite ion gyration (Vandakurov [3]), has the components

$$
\begin{gathered}
P_{x x}=0, \quad P_{x y}=P_{y x} \\
=-2 \rho_{0} v_{0}\left(\frac{\partial w}{\partial x}+\frac{\partial u}{\partial z}\right), \quad P_{x z} \\
=P_{z x}=2 \rho_{0} v_{0}\left(\frac{\partial u}{\partial y}+\frac{\partial v}{\partial x}\right), \\
P_{y y}=-\rho_{0} v_{0}\left(\frac{\partial v}{\partial z}+\frac{\partial w}{\partial y}\right), \quad P_{y z}=P_{z y} \\
=\rho_{0} v_{0}\left(\frac{\partial v}{\partial y}-\frac{\partial w}{\partial z}\right), P_{z z} \\
=\rho_{0} v_{0}\left(\frac{\partial v}{\partial z}+\frac{\partial w}{\partial y}\right) .
\end{gathered}
$$

Here $\rho_{0} v_{0}=\frac{N T}{4 \omega_{H}}$, where $N, T$ and $\omega_{H}$ denote respectively the number density, the ion temperature and the ion gyration frequency.

\section{Dispersion Relation}

Analyzing the disturbances into normal modes, we assume that the perturbation quantities are of the form

$$
\begin{aligned}
& {\left[w, \theta, h_{z}, \zeta, \xi\right]} \\
& =[W(z), \Theta(z), K(z), Z(z), X(z)] \exp \left(i k_{x} x\right. \\
& \left.+i k_{y} y+n t\right),
\end{aligned}
$$

where $k_{x}, k_{y}$ are the wave numbers in the $x-$ and $y-$ directions respectively, $k=$ $\left(k_{x}^{2}+k_{y}^{2}\right)^{1 / 2}$ is the resultant wave number and $n$ is, in general, a complex constant.

Expressing the coordinates $x, y, z$ in the new unit of length $d$ and letting $a=k d, \sigma=\frac{n d^{2}}{v}, p_{1}=$ $v / \kappa, p_{2}=v / \eta$, and $D=\frac{d}{d z}$; Equations (1) $-(5)$ using (8) and expression (9), give 


$$
\begin{gathered}
\left(\frac{\sigma}{\varepsilon}+\frac{1}{P_{l}}\right)\left(D^{2}-a^{2}\right) W+\frac{g \alpha d^{2}}{v} a^{2} \Theta \\
-\frac{i k_{x} H d^{2}}{4 \pi \rho_{0} v}\left(D^{2}-a^{2}\right) K \\
-\left(\frac{i k_{x} v_{0} d^{2}}{v}\right)\left(D^{2}-a^{2}+3 \frac{k_{x}^{2}}{k^{2}} a^{2}\right) Z \\
=0, \\
\left(\frac{\sigma}{\varepsilon}+\frac{1}{P_{l}}\right) Z \\
=-\left(\frac{i k_{x} v_{0}}{v}\right)\left(D^{2}-a^{2}+3 \frac{k_{x}^{2}}{k^{2}} a^{2}\right) W \\
+\frac{i k_{x} H d^{2}}{4 \pi \rho_{0} v} X, \quad=-\frac{i k_{x} H d^{2}}{\varepsilon \eta} W \\
\left(D^{2}-a^{2}-p_{2} \sigma\right) K \\
+\frac{i k_{x} H d^{2}}{4 \pi N e \eta} X \\
\quad-\frac{i k_{x} H}{4 \pi N e \eta}\left(D^{2}-a^{2}\right) K, \\
\left(D^{2}-a^{2}-p_{2} \sigma\right) X \quad\left(\frac{i k_{x} H d^{2}}{\varepsilon \eta} Z\right. \\
\left(D^{2}-a^{2}-E p_{1} \sigma\right) \Theta=-\left(\frac{\beta d^{2}}{\kappa}\right) W .
\end{gathered}
$$

Eliminating $\Theta, K, X$ and $Z$ between equations (10) - (14), we obtain

$$
\begin{aligned}
{\left[( \frac { \sigma } { \varepsilon } + \frac { 1 } { P _ { l } } ) \left(D^{2}\right.\right.} & \left.-a^{2}-p_{2} \sigma\right)^{2} \\
& -Q k_{x}^{2} d^{2}\left(D^{2}-a^{2}-p_{2} \sigma\right) \\
& -M k_{x}^{2} d^{2}\left(\frac{\sigma}{\varepsilon}+\frac{1}{P_{l}}\right)\left(D^{2}\right. \\
& \left.\left.-a^{2}\right)\right]\left[( \frac { \sigma } { \varepsilon } + \frac { 1 } { P _ { l } } ) ( D ^ { 2 } - a ^ { 2 } ) \left(D^{2}\right.\right. \\
& \left.\left.-a^{2}-E p_{1} \sigma\right)-R a^{2}\right] W \\
& -Q k_{x}^{2} d^{2}\left(D^{2}-a^{2}-E p_{1} \sigma\right)\left(D^{2}\right. \\
& \left.-a^{2}\right)
\end{aligned}
$$

$\left[\left\{\left(\frac{\sigma}{\varepsilon}+\frac{1}{P_{l}}\right)\left(D^{2}-a^{2}-p_{2} \sigma\right)-Q k_{x}^{2} d^{2}\right\}\right.$

$\left.-2 M^{1 / 2} N^{1 / 2} k_{x}^{2} d^{2}\left(D^{2}-a^{2}+3 \frac{k_{x}^{2}}{k^{2}} a^{2}\right)\right] W$

$-N k_{x}^{2} d^{2}\left(D^{2}-a^{2}\right.$

(10)

$\left.-E p_{1} \sigma\right)\left(D^{2}-a^{2}\right.$

$\left.+3 \frac{k_{x}^{2}}{k^{2}} a^{2}\right)^{2}\left[\left(D^{2}-a^{2}-p_{2} \sigma\right)^{2}\right.$

$\left.-M k_{x}^{2} d^{2}\left(D^{2}-a^{2}\right)\right] W$

$=0$,

where $R=\frac{g \alpha \beta d^{4}}{v \kappa}$ is the Rayleigh number, $Q=$ $\frac{H^{2} d^{2}}{4 \pi \rho_{0} v \eta \varepsilon}$ is the modified Chandrasekhar number, $M=\left(\frac{H}{4 \pi N e \eta}\right)^{2}$ is a non-dimensional number accounting for Hall current, $N=\left(\frac{v_{0}}{v}\right)^{2}$ is a nondimensional number accounting for FLR effects and $P_{l}=\frac{k_{1}}{\varepsilon d^{2}}$.

Here we consider the case of plasma layer with two free boundaries and the adjoining medium to be nonconducting. The boundaries are assumed to be perfect conductors of heat. The case of two free boundaries is slightly artificial, except in stellar atmospheres (Spiegel [22]) and in certain geophysical situations where it is most appropriate. However, the case of two free boundaries allows us to obtain analytical solution without affecting the essential features of the problem. The appropriate boundary conditions for the present problem are

$W=\Theta=D^{2} W=D Z=0$ at $z=0$ and 1

$X=0$ and $h_{x}, h_{y}, h_{z}$ are continuous.

Using the boundary conditions (16), it can be shown with the help of equations (10) - (14) that all the even order derivatives of $W$ vanish for $z=0$ and 1 and hence the proper solution of (15) characterizing the lowest mode is

$W=W_{0} \sin \pi z$,

where $W_{0}$ is a constant. Substituting (17) in (15) and letting 


$$
\begin{aligned}
a^{2}=\pi^{2} x, R_{1} & =\frac{R}{\pi^{4}}, Q_{1}=\frac{Q}{\pi^{2}}, k_{x} \\
& =k \cos \theta, i \sigma_{1}=\frac{\sigma}{\pi^{2}} \text { and } P \\
& =\pi^{2} P_{l},
\end{aligned}
$$

we obtain the dispersion relation

$$
\begin{aligned}
R_{1}=\left(\frac{i \sigma_{1}}{\varepsilon}+\frac{1}{P}\right) & \left(\frac{1+x}{x}\right)(1+x \\
& \left.+i E p_{1} \sigma_{1}\right)\left[Q_{1} \cos ^{2} \theta(1+x)(1\right. \\
& +x \\
& \left.+i E p_{1} \sigma_{1}\right)\left\{\left(\frac{i \sigma_{1}}{\varepsilon}+\frac{1}{P}\right)(1+x\right. \\
& \left.\left.+i p_{2} \sigma_{1}\right)+Q_{1} x \cos ^{2} \theta\right\} \\
& +2 M^{1 / 2} N^{1 / 2} Q_{1}\left(\cos ^{4} \theta\right) x(1 \\
& +x)\left(1+x+i E p_{1} \sigma_{1}\right)(1+x \\
& \left.-3 x \cos ^{2} \theta\right) \\
& +N \cos ^{2} \theta(1+x \\
& \left.+i E p_{1} \sigma_{1}\right)(1+x \\
& \left.-3 x \cos ^{2} \theta\right)^{2}\left\{\left(1+x+i p_{2} \sigma_{1}\right)^{2}\right. \\
& \left.\left.+M x(1+x) \cos ^{2} \theta\right\}\right]\left[\left(\frac{i \sigma_{1}}{\varepsilon}\right.\right. \\
& \left.+\frac{1}{P}\right)\left(1+x+i p_{2} \sigma_{1}\right)^{2} \\
& +Q_{1} x \cos ^{2} \theta\left(1+x+i p_{2} \sigma_{1}\right) \\
& +M x(1+x)\left(\frac{i \sigma_{1}}{\varepsilon}\right. \\
& \left.\left.+\frac{1}{P}\right) \cos ^{2} \theta\right]^{-1} .
\end{aligned}
$$

\section{Important Theorems Discussion}

Theorem 1: The system is stable or unstable.

Proof: Multiplying equation (10) by $W^{*}$, the complex conjugate of $W$, integrating over the range of $z$ and using equations (11) - (14) together with boundary conditions (16), we obtain

$$
\begin{aligned}
& \left(\frac{\sigma}{\varepsilon}+\frac{1}{P_{l}}\right) I_{1}+\frac{\varepsilon \eta}{4 \pi \rho_{0} v}\left(I_{4}+p_{2} \sigma^{*} I_{5}\right) \\
& +\frac{\varepsilon \eta d^{2}}{4 \pi \rho_{0} v}\left(I_{6}+p_{2} \sigma I_{7}\right)+d^{2}\left(\frac{\sigma^{*}}{\varepsilon}+\frac{1}{P_{l}}\right) I_{8} \\
& =\frac{g \alpha \kappa a^{2}}{v \beta}\left(I_{2}+E p_{1} \sigma^{*} I_{3}\right),
\end{aligned}
$$

where

$$
\begin{gathered}
I_{1}=\int_{0}^{1}\left(|D W|^{2}+a^{2}|W|^{2}\right) d z, \quad I_{2}= \\
\int_{0}^{1}\left(|D \Theta|^{2}+a^{2}|\Theta|^{2}\right) d z, \quad I_{3}=\int_{0}^{1}|\Theta|^{2} d z, \\
I_{4}=\int_{0}^{1}\left(\left|D^{2} K\right|^{2}+2 a^{2}|D K|^{2}+a^{4}|K|^{2}\right) d z, \quad I_{5} \\
=\int_{0}^{1}\left(|D K|^{2}+a^{2}|K|^{2}\right) d z, \\
\begin{aligned}
I_{6}=\int_{0}^{1}\left(|D X|^{2}+\right. & \left.a^{2}|X|^{2}\right) d z, \quad I_{7} \\
& =\int_{0}^{1}|X|^{2} d z, \quad I_{8} \\
& =\int_{0}^{1}|Z|^{2} d z,
\end{aligned}
\end{gathered}
$$

which are all positive definite. Putting $\sigma=\sigma_{r}+$ $i \sigma_{i}$ where $\sigma_{r}, \sigma_{i}$ are real and equating the real and imaginary parts of equation (19), we obtain

$$
\begin{aligned}
& \sigma_{r}\left[\frac{I_{1}}{\varepsilon}+\frac{\varepsilon \eta}{4 \pi \rho_{0} v} p_{2} I_{5}+\frac{\varepsilon \eta d^{2}}{4 \pi \rho_{0} v} p_{2} I_{7}+\frac{d^{2}}{\varepsilon} I_{8}\right. \\
& \left.-\frac{g \alpha \kappa a^{2}}{v \beta} E p_{1} I_{3}\right] \\
& =-\left[\frac{I_{1}}{P_{l}}+\frac{\varepsilon \eta}{4 \pi \rho_{0} v}\left(I_{4}+d^{2} I_{6}\right)+\frac{d^{2}}{P_{l}} I_{8}\right. \\
& \left.-\frac{g \alpha \kappa a^{2}}{v \beta} I_{2}\right],
\end{aligned}
$$

and

$$
\begin{aligned}
\sigma_{i}\left[\frac{I_{1}}{\varepsilon}+\frac{\varepsilon \eta p_{2}}{4 \pi \rho_{0} v} v\right. & \left(d^{2} I_{7}-I_{5}\right)-\frac{d^{2}}{\varepsilon} I_{8} \\
+ & \left.\frac{g \alpha \kappa a^{2}}{v \beta} E p_{1} I_{3}\right]=0 .
\end{aligned}
$$

It is evident from equation (21) that $\sigma_{r}$ may be positive or negative i.e. there may be instability or stability in the system through porous medium in the presence of finite Larmor radius, Hall effects and magnetic field.

Theorem 2: The modes may be oscillatory or non-oscillatory.

Proof: Equation (22) yields that $\sigma_{i}$ may be zero or non-zero, which means that the modes may be non-oscillatory or oscillatory. In the absence of 
magnetic field (and hence absence of FLR and Hall effects), equation (22) reduces to

$$
\sigma_{i}\left[\frac{I_{1}}{\varepsilon}+\frac{g \alpha \kappa a^{2}}{v \beta} E p_{1} I_{3}\right]=0 \text {, }
$$

and the terms in brackets are positive definite. Thus $\sigma_{i}=0$, which means that oscillatory modes are not allowed and the principle of exchange of stabilities is satisfied. The oscillatory modes are introduced due to the presence of magnetic field (and hence the presence of FLR and Hall effects) which were non-existent in their absence.

Theorem 3: The system is stable for $\frac{g \alpha \kappa}{v \beta} \leq \frac{27 \pi^{4}}{4}$ and under the condition $\frac{g \alpha \kappa}{v \beta}>\frac{27 \pi^{4}}{4}$, the system becomes unstable.

Proof: From equation (22), it is clear that $\sigma_{i}$ is zero when the quantity multiplying it is not zero and arbitrary when this quantity is zero.

If $\sigma_{i} \neq 0$, then equation (22) gives

$$
\begin{aligned}
\frac{I_{1}}{\varepsilon}+\frac{\varepsilon \eta d^{2}}{4 \pi \rho_{0} v} p_{2} I_{7} & \\
& =\frac{\varepsilon \eta}{4 \pi \rho_{0} v} p_{2} I_{5}+\frac{d^{2}}{\varepsilon} I_{8} \\
& -\frac{g \alpha \kappa a^{2}}{v \beta} E p_{1} I_{3}
\end{aligned}
$$

Substituting this in equation (21), we get

$$
\begin{aligned}
\frac{2 \sigma_{r} I_{1}}{\varepsilon}+\frac{1}{P_{l}} I_{1}+ & \frac{\varepsilon \eta}{4 \pi \rho_{0} v} I_{4}+\frac{\varepsilon \eta}{4 \pi \rho_{0} v} d^{2} I_{6} \\
& +\frac{\sigma_{r} \varepsilon \eta d^{2}}{2 \pi \rho_{0} v} p_{2} I_{7}+\frac{d^{2}}{P_{l}} I_{8} \\
& =\frac{g \alpha \kappa a^{2}}{v \beta} I_{2} .
\end{aligned}
$$

Equation (24) on using Rayleigh-Ritz inequality gives

$$
\begin{aligned}
& \frac{\left(\pi^{2}+a^{2}\right)^{3}}{a^{2}} \int_{0}^{1}|W|^{2} d z \\
& +\frac{\left(\pi^{2}+a^{2}\right)}{a^{2}}\left\{d^{2}\left(\frac{1}{P_{l}}\right) I_{8}+\frac{\sigma_{r} \varepsilon \eta d^{2}}{2 \pi \rho_{0} v} p_{2} I_{7}\right. \\
& \left.+\frac{\varepsilon \eta}{4 \pi \rho_{0} v} d^{2} I_{6}+\frac{\varepsilon \eta}{4 \pi \rho_{0} v} I_{4}+\frac{1}{P_{i}} I_{1}+\frac{2 \sigma_{r}}{\varepsilon} I_{1}\right\} \\
& \leq \frac{g \alpha \kappa}{\beta v} \int_{0}^{1}|W|^{2} d z .
\end{aligned}
$$

$$
\begin{aligned}
& {\left[\frac{27 \pi^{4}}{4}-\frac{g \alpha \kappa}{v \beta}\right] \int_{0}^{1}|W|^{2} d z} \\
& +\frac{\left(\pi^{2}+a^{2}\right)}{a^{2}}\left\{d^{2}\left(\frac{1}{P_{l}}\right) I_{8}+\frac{\sigma_{r} \varepsilon \eta d^{2}}{2 \pi \rho_{0} v} p_{2} I_{7}\right. \\
& \left.+\frac{\varepsilon \eta}{4 \pi \rho_{0} v} d^{2} I_{6}+\frac{\varepsilon \eta}{4 \pi \rho_{0} v} I_{4}+\frac{1}{P_{i}} I_{1}+\frac{2 \sigma_{r}}{\varepsilon} I_{1}\right\} \\
& \leq 0,
\end{aligned}
$$

since minimum value of $\frac{\left(\pi^{2}+a^{2}\right)^{3}}{a^{2}}$ with respect to $a^{2}$ is $\frac{27 \pi^{4}}{4}$.

Now, let $\sigma_{r} \geq 0$, we necessary have from (26) that

$\frac{g \alpha \kappa}{v \beta}>\frac{27 \pi^{4}}{4}$.

Hence, if

$\frac{g \alpha \kappa}{v \beta} \leq \frac{27 \pi^{4}}{4}$,

then $\sigma_{r}<0$. Therefore, the system is stable. Thus, under condition (28), the system is stable and under condition (27) the system becomes unstable.

Theorem 4: For stationary convection

FLR may have a stabilizing or destabilizing effect, but a completely stabilizing one for a certain wave-number range $\cos \theta<\left\{\frac{1+x}{3 x}\right\}^{1 / 2}$.

(II) In the absence of Hall currents, FLR always has a stabilizing effect.

(III) The Hall currents may have a stabilizing or destabilizing effect but completely stabilizes the wavenumber range $\cos \theta<\left\{\frac{1+x}{3 x}\right\}^{1 / 2}$ if $\frac{k_{1}}{\rho_{0} v}>\frac{\varepsilon^{2}}{4 \pi^{3} \eta N^{2} e^{2}}$.

(IV) The medium permeability always has a destabilizing effect on the system for stationary convection.

Proof: When the instability sets in as stationary convection, the marginal state will be characterized by $\sigma=0$. Putting $\sigma=0$, the dispersion relation (18) reduces to

Therefore, it follows from relation (25) that 
$R_{1}$

$=\frac{(1+x)^{2}}{x P}$

$+\left[Q_{1}(1+x) \cos ^{2} \theta\left(\frac{1+x}{P}+Q_{1} x \cos ^{2} \theta\right)\right.$

$+2 M^{1 / 2} N^{1 / 2} Q_{1}\left(\cos ^{4} \theta\right) x\left(1+x-3 x \cos ^{2} \theta\right)(1$

$+x)$

$+N \cos ^{2} \theta(1+x)\left(1+x-3 x \cos ^{2} \theta\right)^{2}(1+x$

$\left.\left.+M x \cos ^{2} \theta\right)\right]\left[\frac{1+x}{P}+Q_{1} x \cos ^{2} \theta\right.$

$\left.+\frac{M x \cos ^{2} \theta}{P}\right]^{-1}$,

which expresses the modified Rayleigh number $R_{1}$ as a function of the dimensionless wave number $x$ and the parameters $Q_{1}, M, N$ and $P$. To study the effects of finite Larmor radius, Hall currents and medium permeability, we examine the nature of $d R_{1} / d N, d R_{1} / d M$, and $d R_{1} / d P$ analytically.

(I) It follows from equation (29),

$$
\begin{aligned}
& \frac{d R_{1}}{d N} \\
& =\left[(1+x)\left(1+x-3 x \cos ^{2} \theta\right)^{2}(1+x\right. \\
& \left.+M x \cos ^{2} \theta\right) \cos ^{2} \theta \\
& +\left(\frac{M}{N}\right)^{1 / 2} Q_{1} x(1+x)(1+x \\
& \left.\left.-3 x \cos ^{2} \theta\right) \cos ^{4} \theta\right]\left[\frac{1+x}{P}+Q_{1} x \cos ^{2} \theta\right. \\
& \left.+\frac{M x \cos ^{2} \theta}{P}\right]^{-1},
\end{aligned}
$$

which is positive for $(1+x)>3 x \cos ^{2} \theta$ i.e. for the wave number range satisfying

$$
\cos \theta<\left\{\frac{1+x}{3 x}\right\}^{1 / 2}
$$

This shows that FLR has a stabilizing effect for the wave number range (31). Thus, in the presence of Hall effects on the thermal instability in porous medium, the FLR may have stabilizing or destabilizing effects but completely stabilizes the wave number range (31).

(II) In the absence of Hall currents $(\mathrm{M} \rightarrow 0)$, equation $(30)$ reduces to $\frac{d R_{1}}{d N}$

$=\left[(1+x)\left(1+x-3 x \cos ^{2} \theta\right)^{2}(1\right.$

$\left.+x) \cos ^{2} \theta\right]\left[\frac{1+x}{P}\right.$

$\left.+Q_{1} x \cos ^{2} \theta\right]^{-1}$

which is always positive, hence in the absence of Hall currents, FLR always has a stabilizing effect.

(III) Equation (29) also yields

$$
\begin{aligned}
& \frac{d R_{1}}{d M} \\
& =\left[N x ( 1 + x ) ( 1 + x - 3 x \operatorname { c o s } ^ { 2 } \theta ) ^ { 2 } \operatorname { c o s } ^ { 4 } \theta \left(\frac{1+x}{P}\right.\right. \\
& \left.+Q_{1} x \cos ^{2} \theta\right) \\
& +\left(\frac{N}{M}\right)^{1 / 2} \frac{Q_{1}}{P} x(1+x)^{2}(1+x \\
& \left.-3 x \cos ^{2} \theta\right) \cos ^{4} \theta \\
& +Q_{1} x^{2} \cos ^{6} \theta(1+x \\
& \left.-3 x \cos ^{2} \theta\right) \frac{1}{P}\left(\frac{N}{M}\right)^{1 / 2}\left(P Q_{1}\right. \\
& -M)]\left[\frac{1}{P}\left(1+x+M x \cos ^{2} \theta\right)\right. \\
& \left.+Q_{1} x \cos ^{2} \theta\right]^{-2},
\end{aligned}
$$

which is positive if $P Q_{1}>M$ and $(1+x)>$ $3 x \cos ^{2} \theta$ i.e. if

$$
\frac{k_{1}}{\rho_{0} v}>\frac{\varepsilon^{2}}{4 \pi^{3} \eta N^{2} e^{2}} \text { and } \cos \theta<\left(\frac{1+x}{3 x}\right)^{1 / 2} \text {. }
$$

In the presence of FLR and Hall effects on the thermal instability in porous medium, the Hall currents may have stabilizing or destabilizing effect but completely stabilizes the wave number range $(31)$ if

$$
\frac{k_{1}}{\rho_{0} v}>\frac{\varepsilon^{2}}{4 \pi^{3} \eta N^{2} e^{2}}
$$

Hence the result.

(IV) It is evident from equation (29) that 


$$
\begin{aligned}
\frac{d R_{1}}{d P}=-[(1+ & x)^{4} \\
& +2 x(1+x)^{3}\left(P Q_{1}+M\right) \cos ^{2} \theta \\
& +x^{2} M(1+x)^{2}(M \\
& \left.+2 P Q_{1}\right) \cos ^{4} \theta \\
& +2 P^{2} Q_{1}^{2} x^{2}(1 \\
& \left.+x)^{2} \cos ^{4} \theta\right]\left[P^{2} x\{1+x\right. \\
& \left.\left.\left.+x \cos ^{2} \theta\left(P Q_{1}+M\right)\right\}\right]^{-2}, \quad 34\right)
\end{aligned}
$$

which means that medium permeability has a destabilizing effect.

\section{Conclusions}

The thermal convection of a plasma in a porous medium in the presence of a finite Larmor radius (FLR) and Hall effects is considered in the present paper. The main conclusions from the analysis of this paper are as follows:

- It is found that magnetic field (and hence the presence of FLR and Hall effects) introduce oscillatory modes in the system which were non-existent in their absence.

- It is observed that system is stable for $\frac{g \alpha \kappa}{v \beta} \leq \frac{27 \pi^{4}}{4}$ and under the condition $\frac{g \alpha \kappa}{v \beta}>\frac{27 \pi^{4}}{4} \quad$, the system becomes unstable.

- For the case of stationary convection:

$>$ FLR may have a stabilizing or destabilizing effect, but a completely stabilizing one for a certain wave-number range $\cos \theta<\left\{\frac{1+x}{3 x}\right\}^{1 / 2}$.

$>$ In the absence of Hall currents, FLR always has a stabilizing effect.

$>$ The Hall currents may have a stabilizing or destabilizing effect but completely stabilizes the wave-number range $\cos \theta<$ $\left\{\frac{1+x}{3 x}\right\}^{1 / 2}$ if $\frac{k_{1}}{\rho_{0} v}>\frac{\varepsilon^{2}}{4 \pi^{3} \eta N^{2} e^{2}}$.

The medium permeability always has a destabilizing effect on the system for stationary convection.

\section{References}

[1] Chandrasekhar, S., Hydrodynamic and hydromagnetic stability, Dover Publication, New York, 1981.

[2] Jukes, J.D., Gravitational resistive instabilities in plasma with finite Larmor radius, Phys. Fluids, Vol. 7, 1964, pp. 52-58.

[3] Vandakurov, Ju. V., On the theory of stability of a plasma in a strong longitudinal magnetic field, variable along the axis of symmetry, Prikl. Mat. Mekh., Vol. 28, 1964, pp. 69-74.

[4] Sharma, R.C. and Prakash, K., Thermal instability in plasma with finite Larmor radius, $Z$. Naturforsch., Vol. 30a, 1975, pp. 461-465.

[5] Melchior, H. and Popowich, M., Effect of the finite ion Larmor radius on the Kelvin-Helmholtz instability, Phys. Fluids, Vol. 11, 1968, pp. 458465.

[6] Singh, S. and Hans, H., Magnetic viscosity and the superposed fluids in a magnetic field, Nucl. Fusion, Vol. 6, 1966, pp. 6-11.

[7] Sharma, R.C., Finite Larmor radius and Hall effects on thermal instability of a rotating plasma, Phys. Fluids, Vol. 15, 1972, pp. 18221826.

[8] Joseph, D.D., Stability of Fluid Motions, Vol. 1 and 2, Springer-Verlag, Berlin, 1976.

[9] McDonnel, J.A.M., Cosmic Dust, John Wiley and Sons, Toronto, p. 330, 1978.

[10] Lapwood, E.R., Convection of a fluid in a porous medium, Proc. Camb. Soc., Vol. 44, 1948, pp. 508-554.

[11] Wooding, R.A., Rayleigh instability of a thermal boundary layer in flow through a porous medium, J. Fluid Mech., Vol. 9, 1960, pp. 183192.

[12] Roberts, K.V. and Taylor, J.B., Magnetohydrodynamics equations for finite Larmor radius, Phys Rev. Lett., Vol. 8, 1962, pp. 197-198.

[13] Rosenbluth, M. N., Krall, N. and Rostoker, N., Finite Larmor radius stabilization of "weakly" unstable confined plasmas, Nucl. Fusion Suppl., Pt. 1, 1962, pp. 143-150. 
[14] Hernegger, F., Effect of collisions and gyroviscosity on gravitational instability in a two-component plasma. J. Plasma Phys., Vol. 8, 1972, pp. 393-400.

[15] Sharma, R. C., Gravitational instability of a rotating plasma, Astrophys. Space Sci., Vol. 29, 1974, pp. L1-L4.

[16] Ariel, P. D., Effect of finite Larmor radius on the gravitational instability of a conducting plasma layer of finite thickness surrounded by a non-conducting matter, Astrophys. Space Sci., Vol. 141, 1988, pp. 141-149.

[17] Vaghela, D. S. and Chhajlani, R. K., Magnetogravitational stability of resistive plasma through porous medium with thermal conduction and FLR corrections, Contrib. Plasma Phys., Vol. 29, 1989, pp. 77-89.

[18] Bhatia, P. K. and Chhonkar, R. P. S., Larmor Radius effects on the instability of a rotating layer of a self-gravitating plasma, Astrophys. Space Sci., Vol. 115, 1985, pp. 327344.
[19] Vyas, M. K. and Chhajlani, R. K., Influence of finite Larmor radius with finite electrical and thermal conductivities on the gravitational instability of a magnetized rotating plasma through a porous medium, Contrib. Plasma Phys., Vol. 30, 1990, pp. 315-328.

[20] Kaothekar, S. and Chhajlani, R. K., Jeans instability of self-gravitating rotating radiative plasma with unite Larmor radius corrections, $J$. Phys. Conf. Series, Vol. 534, 2014, pp. 012065.

[21] Kumar, P. and Mohan, H., On the stability of composite plasma in porous medium, Ikonion J. Maths. Vol. 2(1), 2020, pp. 21-27.

[22] Spiegel, E.A., Convective instability in a compressible atmosphere, Astrophys. J., Vol. 141, 1965, pp. 1068-1070.

\section{Creative Commons Attribution License 4.0 (Attribution 4.0 International, CC BY 4.0)}

This article is published under the terms of the Creative Commons Attribution License 4.0

https://creativecommons.org/licenses/by/4.0/deed.en_US 